\title{
Lung transplantation for coronavirus disease 2019 (COVID-19): The who, what, where, when, and why
}

Lara Schaheen, MD, Ross M. Bremner, MD, PhD, Rajat Walia, MD, and Michael A. Smith, MD

Coronavirus disease 2019 (COVID-19) has caused a global pandemic of unprecedented magnitude in the last century. As of March 2021, there have been more than 128 million confirmed cases of COVID-19, leading to the death of more than 2.5 million people worldwide. ${ }^{1}$ Although the symptoms associated with COVID-19 are diverse, the majority of serious consequences from COVID-19 involve pneumonia. ${ }^{2}$

Early data demonstrated that severe acute respiratory syndrome coronavirus 2 (SARS-CoV-2) infection progressed to severe respiratory failure and acute respiratory distress syndrome (ARDS) in up to $42 \%$ of hospitalized patients. ${ }^{3}$ Despite optimized supportive care, mortality rates of patients with COVID-19 requiring mechanical ventilation are between $20 \%$ and $40 \%{ }^{4,5}$ Several early studies reported a mortality rate of up to $60 \%$ in patients with severe COVID-19-associated ARDS, greater than the $30 \%$ to $45 \%$ mortality rate reported with other causes of ARDS..$^{6-9}$

The mechanisms through which SARS-CoV-2 causes lung injury are unknown but likely multifactorial, with contributions from the resulting cytokine release syndrome, ventilator-induced lung injury, drug-induced pulmonary toxicity, secondary nosocomial pneumonia, and thrombosis. At present, the long-term pulmonary consequences of COVID-19-associated ARDS and the spectrum of lung recoverability remain unknown.

Although lung transplantation (LTx) is a life-saving treatment for end-stage lung disease (ESLD), its role in patients with ARDS remains controversial. Results from individual case reports and a retrospective single-center series have demonstrated promising early survival, but these studies are limited by small patient numbers, a wide spectrum of causes leading to ARDS, and the absence of long-term outcomes. To date, the role and timing of LTx for ARDS remains unclear, as there is great uncertainty in determining the reversibility of lung injury and the potential for lung recovery. A recent review of the United Network for Organ Sharing (UNOS) database identified a total of 63 patients

\footnotetext{
From the St Joseph's Hospital and Medical Center, Norton Thoracic Institute, Phoenix, Ariz.

Received for publication April 5, 2021; revisions received June 1, 2021; accepted for publication June 10, 2021; available ahead of print July 6, 2021.

Address for reprints: Michael A. Smith, MD, St Joseph's Hospital and Medical Center, Norton Thoracic Institute, 500 W. Thomas Rd, Suite 500, Phoenix, AZ 85013 (E-mail: Michael.Smith001@DignityHealth.org).

J Thorac Cardiovasc Surg 2022;163:865-8

$0022-5223 / \$ 36.00$

Copyright (c) 2021 by The American Association for Thoracic Surgery https://doi.org/10.1016/j.jtcvs.2021.06.061
}

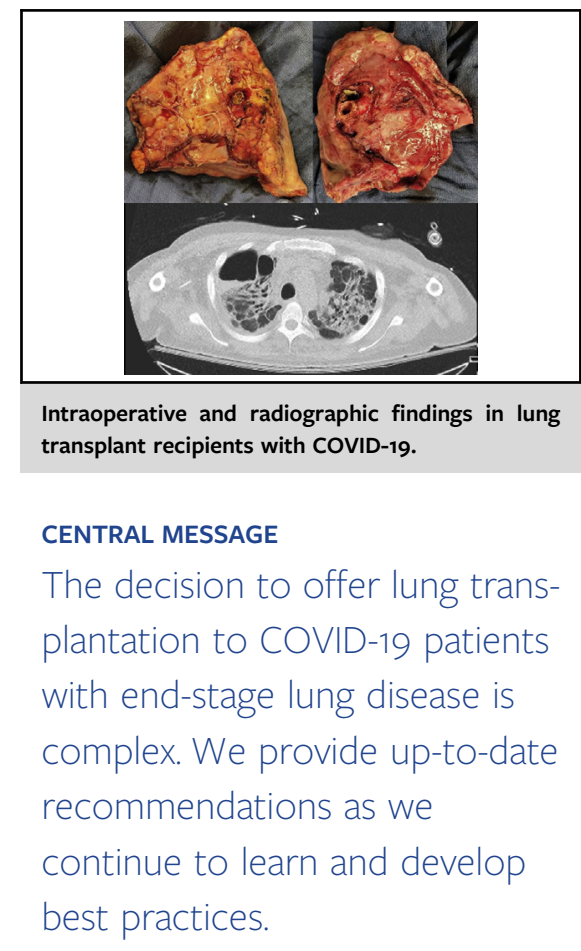

See Commentaries on pages 869 and 870.

who were listed for LTx with a primary diagnosis of nonCOVID-19-associated ARDS from May 2005 through December 2018. Of the 63 patients waitlisted, 39 patients ultimately received a lung transplant. Of the remaining 24 patients who were waitlisted but did not receive a lung transplant, $16.7 \%$ were removed due to clinical improvement. ${ }^{10}$ Optimizing the potential for native lung recovery and avoiding a lung transplant is likely best practice, considering the limited supply of acceptable donor lungs in comparison with the number of patients currently awaiting a lung transplant as well as the less-than-desirable longterm survival expectations for LTx recipients. Thus, it is our best practice opinion that LTx is considered only when sufficient time has elapsed to exclude meaningful lung recovery.

Venovenous extracorporeal membrane oxygenation (ECMO) has historically been considered a last-line therapy for patients with refractory hypoxemia or hypercapnia when conventional management has failed. However, ECMO has progressed remarkably over recent years through advancements in circuit design, single-site cannulation, awake status, and patient mobilization. These clinical advancements 
and growing institutional experience have undoubtedly contributed to the remarkable increase in the number of patients treated with ECMO and the improvement in outcomes observed when ECMO is used to treat patients with severe ARDS. ${ }^{11}$ In support of this, a post-hoc Bayesian analysis of data from the ECMO to Rescue Lung Injury in Severe ARDS (EOLIA) trial demonstrated a high likelihood of an ECMO survival benefit for severe ARDS. ${ }^{12}$

These data have contributed to a significant increase in the number of patients treated with ECMO in recent years; however, data regarding the correct duration of support and time needed for evaluation, treatment, and recovery remain lacking. Previous studies have shown that lungs severely injured by viral or bacterial pneumonia leading to severe ARDS can recover to support life after weeks to months on ECMO. In a review of the long-term outcomes of patients treated for H1N1-associated ARDS, patients had mild disabilities 1 year after discharge from the intensive care unit; most of them had no demonstrable weakness and had returned to work. ${ }^{13}$ We can apply the understanding that we have gained from this impressive progress to the current pandemic.

Accordingly, international organizations and experts in the field recommended ECMO support for patients who are critically ill with COVID-19. ${ }^{14,15}$ Schmidt and colleagues ${ }^{16}$ demonstrated that ECMO allowed for the use of protective ventilation with decreased plateau pressure, respiratory rate, and tidal volume in patients with COVID-19-associated ARDS. They also demonstrated that the estimated 60-day survival of these patients rescued by ECMO was similar to that of recent studies on ECMO for severe ARDS from other causes.

Unfortunately, some patients with ARDS will progress to the fibrotic phase, preventing them from successful separation from ECMO and leaving them with irreversible ESLD. Ichikado and colleagues ${ }^{17}$ demonstrated that the presence of extensive fibroproliferative changes seen on computed tomography images may serve as an independent predictor of poor prognosis in patients with ARDS. Chung and colleagues ${ }^{18}$ demonstrated that computed tomography findings of $>80 \%$ of lung involvement, a right atrium to left atrium ratio $>1$, and varicoid bronchiectasis portended the greatest risk of mortality. Findings of extensive honeycombing, reticular opacities, and traction bronchiectasis may help identify patients who have a lower chance of recovery and should be considered for LTx.

The uncertainty of these patients' long-term outcomes, lack of clarity on the intended treatment direction, whether bridge to recovery or bridge to transplant (BTT) will be successful, and the optimal duration of support remain an evolving challenge. In patients who have advanced findings of irreversible pulmonary fibrosis following ARDS, ECMO has proven to be useful to bridge patients to LTx. We have previously published our experience with ECMO BTT in
$13 \%$ of our lung transplant recipients between January 2015 and December 2015, with 100\% 1-year survival in the ECMO BTT cohort. ${ }^{19}$ In addition, another retrospective analysis of the UNOS database reported that a total of 119 recipients were bridged to LTx with ECMO between January 2000 and December 2011. The authors demonstrated that age served as a predictor of 1-year mortality. ${ }^{20}$

Modern ECMO devices have expanded the therapeutic possibilities available for patients with end-stage lung failure-in many instances allowing a patient to remain awake and able to participate in physical therapy. Hoetzenecker and the Toronto group ${ }^{21}$ first introduced the idea of awake ECMO as a bridge to transplant in 2008. In 2017, they reported that an impressive $37 \%$ of all patients undergoing ECMO therapy while awaiting transplantation at their institution were mobilized. Schechter and colleagues ${ }^{15}$ recently published a UNOS database analysis focusing on awake extracorporeal life support bridging. In a cohort of approximately 12,500 LTx recipients, $65(0.52 \%)$ were bridged with ECMO in an awake, spontaneously breathing setting, and $119(0.96 \%)$ required ECMO and mechanical ventilation during bridging. The awake ECMO group experienced significantly better outcomes, with a 3 -year survival of $64.5 \% .^{15}$ In a comparison of patients undergoing BTT who successfully reached transplantation with those who died during the bridging period, an awake status was an independent predictor of survival. The improved outcomes and survival in awake and/or mobilized BTT patients may provide insight into the LTx candidacy for patients with COVID-19-associated ARDS.

Unlike other causes of ESLD, COVID-19-associated ARDS presents a unique challenge due to the unknown effects of the true viral status and the potential for recurrence in the new lungs. In some patients, persistently positive SARS-CoV-2 testing may further complicate the clinician's decision to proceed with lung transplant evaluation. Due to these unanswered questions, Cypel and Keshavjee ${ }^{22}$ recommended that multiple lower respiratory tract samples be tested to confirm a negative infection status before listing for LTx. To further muddy the waters, recent studies have demonstrated that the presence of viral particles on polymerase chain reaction (PCR) testing does not necessarily correlate to the presence of infectious virus. Bullard and colleagues $^{23}$ recently tested infectivity of SARS-CoV-2positive reverse-transcription PCR samples and demonstrated that infectivity was observed in samples only from patients with a duration of symptoms less than 8 days and a reverse-transcription PCR cycle threshold value $<24$. Until our understanding of the relationship between positive SARS-CoV-2 test results and infectivity is complete, we recommend that LTx is delayed until recipients are known to be without active SARS-CoV-2 virus.

Having reviewed COVID-19 disease progression, the role of ECMO and LTx in ARDS, and outcomes of 
ECMO BTT, we are led to the challenging discussion regarding LTx candidacy for patients with ESLD following COVID-19. The patients with ESLD from COVID-19 ARDS-related fibrosis are particularly challenging, as we do not definitely know the right time for LTx or how long to wait for patients to recover before moving forward with LTx. The development of other organ dysfunction, such as right heart failure, should be heavily considered in determining the optimal time to move forward with LTx evaluation. This challenge is further amplified by the prolonged disease course that some of these patients follow, with extraordinary use of resources. Even patients who have definitely entered the fibrotic phase of lung disease present us with new challenges in rendering them acceptable to move forward with LTx. This is especially true when considering the perils of a limited organ supply and other ill patients who exist within each of our recipient lists. To this end, we offer several recommendations based on our growing experience:

1. The patient should fulfill standard criteria for LTx. ${ }^{24}$

2. The patient should be younger than 65 years or extremely carefully selected if older than 65 years.

3. LTx should be considered only when sufficient time has elapsed to exclude native lung recovery. In our experience, this is a minimum of 8 weeks.

4. Radiographic findings alone should not be used to determine recoverability but should be correlated with the patient's clinical course.

5. Negative SARS-CoV-2 virology status should be confirmed by bronchoalveolar lavage-repeatedly if necessary.

6. Irreversible concomitant organ failure must be absent.

7. The patient should be able to actively participate in physical rehabilitation.

8. The patient should be able to provide first-person consent to LTx and transfusion.

9. Antibodies should be carefully evaluated due to the likely history of exposure in the critical illness period leading up to listing.

10. The patient should have minimal acute comorbidity.

11. Decisions regarding these patients should be critically re-evaluated on a periodic basis.

We believe that due to the underlying pulmonary hypertension and superimposed infections seen in this population, a bilateral LTx should be performed in appropriate candidates recovering from ESLD due to COVID-19. The induction and antibiotic regimen along with the operation should follow standard procedures. Donor lungs should be sized appropriately in consideration of the restrictive nature of the recipient and any chest size changes that have occurred in the acute illness period. The operation can be particularly challenging in these patients, especially if there have been intrathoracic procedures or other processes during the critical illness phase (eg, chest tubes, thoracoscopy, thoracotomy, empyema). The anesthesiology and operative teams should be prepared to manage the transfusion and hemodynamic requirements, considering the uncertainty of what might be encountered. Intraoperative mechanical circulatory support, such as ECMO or cardiopulmonary bypass, is needed much more frequently in this patient population due to the diffuse nature of the recipient's native lung disease and underlying pulmonary hypertension.

We strongly suggest that patients with ESLD due to COVID-19 are referred to transplant centers with extensive experience in high-risk LTx and ECMO as a BTT. We make this recommendation based on our own experience and that of others. In the recovery period after transplantation, avoiding the specific and uncertain COVID-19-related bacterial, viral, and other opportunistic infections requires the approach of an experienced center that can pivot quickly to keep the patient on a positive postoperative trajectory. The potential volume of patients needing a lung transplant from COVID-19 ARDS, the possibility of an even more limited donor organ supply during a pandemic, and the uncertainty of mid- and long-term outcomes for recipients with ESLD secondary to COVID-19 remain unknown. The list of unanswered questions is remarkable and includes potential infectious sequelae, rejection episodes, other COVID-19-specific morbidities, and potential viral reactivation. What is key is that we acknowledge the dynamic nature of this area and adapt accordingly to new information as it emerges. Finally, we emphasize that we also must not lose sight of other patients on our waitlist who are in desperate need of new lungs.

\section{Conflict of Interest Statement}

The authors reported no conflicts of interest.

The Journal policy requires editors and reviewers to disclose conflicts of interest and to decline handling or reviewing manuscripts for which they may have a conflict of interest. The editors and reviewers of this article have no conflicts of interest.

\section{References}

1. World Health Organization. WHO coronavirus (COVID-19) dashboard. Available at: https://covid19.Who.Int/. Accessed April 1, 2021.

2. Guan WJ, Ni ZY, Hu Y, Liang WH, Ou CQ, He JX, et al. Clinical characteristics of coronavirus disease 2019 in China. N Engl J Med. 2020;382:1708-20.

3. Wu C, Chen X, Cai Y, Xia J, Zhou X, Xu S, et al. Risk factors associated with acute respiratory distress syndrome and death in patients with coronavirus disease 2019 pneumonia in Wuhan, China. JAMA Intern Med. 2020;180:934-43.

4. Horby P, Lim WS, Emberson JR, Mafham M, Bell JL, Linsell L, et al. Dexamethasone in hospitalized patients with COVID-19. N Engl J Med. 2021;384:693-704.

5. Olalla J. Remdesivir for the treatment of COVID-19 - preliminary report. $N$ Engl J Med. 2020;383:993-4.

6. Grasselli G, Zangrillo A, Zanella A, Antonelli M, Cabrini L, Castelli A, et al. Baseline characteristics and outcomes of 1591 patients infected with SARSCoV-2 admitted to ICUs of the Lombardy region, Italy. JAMA. 2020;323: 1574-81. 
7. Wang D, Hu B, Hu C, Zhu F, Liu X, Zhang J, et al. Clinical characteristics of 138 hospitalized patients with 2019 novel coronavirus-infected pneumonia in Wuhan, China. JAMA. 2020;323:1061-9.

8. Yang X, Yu Y, Xu J, Shu H, Xia J, Liu H, et al. Clinical course and outcomes of critically ill patients with SARS-CoV-2 pneumonia in Wuhan, China: a singlecentered, retrospective, observational study. Lancet Respir Med. 2020;8:475-81.

9. Máca J, Jor O, Holub M, Sklienka P, Burša F, Burda M, et al. Past and present ARDS mortality rates: a systematic review. Respir Care. 2017;62:113-22.

10. Harano T, Ryan JP, Chan EG, Noda K, Morrell MR, Luketich JD, et al. Lung transplantation for the treatment of irreversible acute respiratory distress syndrome. Clin Transplant. 2021;35:e14182.

11. Peek GJ, Mugford M, Tiruvoipati R, Wilson A, Allen E, Thalanany MM, et al. Efficacy and economic assessment of conventional ventilatory support versus extracorporeal membrane oxygenation for severe adult respiratory failure (CESAR): a multicentre randomised controlled trial. Lancet. 2009;374:1351-63.

12. Goligher EC, Tomlinson G, Hajage D, Wijeysundera DN, Fan E, Jüni P, et al. Extracorporeal membrane oxygenation for severe acute respiratory distress syndrome and posterior probability of mortality benefit in a post hoc Bayesian analysis of a randomized clinical trial. JAMA. 2018;320:2251-9.

13. Luyt CE, Combes A, Becquemin MH, Beigelman-Aubry C, Hatem S, Brun AL, et al. Long-term outcomes of pandemic 2009 influenza A (H1N1)-associated severe ARDS. Chest. 2012;142:583-92.

14. MacLaren G, Fisher D, Brodie D. Preparing for the most critically ill patients with COVID-19: the potential role of extracorporeal membrane oxygenation. JAMA. 2020;323:1245-6.

15. Schechter MA, Ganapathi AM, Englum BR, Speicher PJ, Daneshmand MA, Davis RD, et al. Spontaneously breathing extracorporeal membrane oxygenation support provides the optimal bridge to lung transplantation. Transplantation. 2016;100:2699-704.

16. Schmidt M, Hajage D, Lebreton G, Monsel A, Voiriot G, Levy D, et al. Extracorporeal membrane oxygenation for severe acute respiratory distress syndrome associated with COVID-19: a retrospective cohort study. Lancet Respir Med. 2020;8:1121-31.

17. Ichikado K, Suga M, Muranaka H, Gushima Y, Miyakawa H, Tsubamoto M, et al. Prediction of prognosis for acute respiratory distress syndrome with thin-section CT: validation in 44 cases. Radiology. 2006;238:321-9.

18. Chung JH, Kradin RL, Greene RE, Shepard JA, Digumarthy SR. Ct predictors of mortality in pathology confirmed ARDS. Eur Radiol. 2011;21:730-7.

19. Todd EM, Biswas Roy S, Hashimi AS, Serrone R, Panchanathan R, Kang P, et al. Extracorporeal membrane oxygenation as a bridge to lung transplantation: a single-center experience in the present era. J Thorac Cardiovasc Surg. 2017; 154:1798-809.

20. Hayanga AJ, Aboagye J, Esper S, Shigemura N, Bermudez CA, D'Cunha J, et al. Extracorporeal membrane oxygenation as a bridge to lung transplantation in the United States: an evolving strategy in the management of rapidly advancing pulmonary disease. J Thorac Cardiovasc Surg. 2015;149:291-6.

21. Hoetzenecker K, Donahoe L, Yeung JC, Azad S, Fan E, Ferguson ND, et al. Extracorporeal life support as a bridge to lung transplantation-experience of a high-volume transplant center. J Thorac Cardiovasc Surg. 2018;155:1316-28.e1.

22. Cypel M, Keshavjee S. When to consider lung transplantation for covid-19. Lancet Respir Med. 2020;8:944-6.

23. Bullard J, Dust K, Funk D, Strong JE, Alexander D, Garnett L, et al. Predicting infectious severe acute respiratory syndrome coronavirus 2 from diagnostic samples. Clin Infect Dis. 2020;71:2663-6.

24. Weill D, Benden C, Corris PA, Dark JH, Davis RD, Keshavjee S, et al. A consensus document for the selection of lung transplant candidates: 2014 —an update from the pulmonary transplantation council of the international society for heart and lung transplantation. J Heart Lung Transplant. 2015;34:1-15.

Key Words: COVID-19, extracorporeal membrane oxygenation, SARS-CoV-2, lung transplantation 